\title{
FUNCTION VALUES AS BOUNDARY INTEGRALS
}

\author{
RICHARD ARENS AND I. M. SINGER
}

1. Introduction. For suitable families $H$ of numerical-valued continuous functions on a topological space $S$ (see 2.4 below) we show that there is a smallest closed subset $B$ of $S$ such that on $B$ every function in the family attains its maximum absolute value. The construction is patterned after that of the "Šilov boundary" ([III] or, more conveniently, [I, p. 80]) where the family of functions has to be an algebra. Algebraically, our families have to be multiplicative semigroups.

We then proceed to show that for each $s \in S$ there is at least one regular Baire measure $m_{s}$ on $B$ such that if $g \in H$ and its reciprocal also belongs to $H$, then $\log |g(s)|=\int_{B} \log |g(b)| m_{s}(d b)$. Our main effort is directed toward finding how this integral representation generalizes for those $g \in H$ whose reciprocal may not exist or at least be absent from $H$. In those cases where $H$ is rich enough to ensure that the measures $m_{\varepsilon}$ are unique for each $s$, we obtain $\log |g(s)|$ $\leqq \int_{B} \log |g(b)| m_{s}(d b)$, which is to say that the geometric mean (relative to $m_{s}$ ) of $|g|$ on $B$ is not less than its value at $s$. This generalizes a classical inequality due to Szegö [II] for regular functions on the disc $S$, where $B$ is the usual boundary.

In the nonunique case we show (see 6.4) that there is for each $g$ at least one measure for which the inequality holds, but there may be some for which it fails.

We intend to published elsewhere a more detailed discussion of these matters for a special type of Banach algebras generalizing more directly the classical case of functions regular on the disc (see \$4). In this case, the boundary is a locally compact abelian group.

2. Existence of the boundary. Let $H$ be a class of real or complex, bounded continuous functions on a topological space $S$. Consider a subset $F$ of $S$ with the properties:

$2.1 F$ is closed,

2.2 for each $h$ in $H,|h(s)|$ attains on $F$ the value $\sup _{s \in S}|h(s)|$; and moreover

2.3 If any subset $F_{1}$ of $S$ has the properties of $F$ in 2.1 and 2.2, then $F_{1}$ contains $F$.

Such a set, if it exists, is evidently unique, and will be denoted by $\partial_{H} S$, and may be called the Silov boundary of $S$ relative to $H$.

Received by the editors December 10, 1953. 
If the class $H$ of functions is replaced by the class of their absolute values, $\partial_{H} S$, as well as its existence, is clearly unaffected. We therefore deal with non-negative real-valued functions only.

2.4 Theorem. Let $S$ be a topological space and $H$ a class of realvalued non-negative continuous functions on $S$ such that

2.41 if $h, j \in H$ then $h j \in H$,

2.42 if $\epsilon>0$ and $h \in H$, the set $\{s ; h(s) \geqq \epsilon\}$ is compact,

2.43 each point $s_{0}$ in $S$ has a basis of neighborhoods of the form

$$
\left\{s ; h_{1}(s)<\epsilon, h_{2}(s)<\epsilon, \cdots, h_{n}(s)<\epsilon\right\},
$$

where $h_{1}, \cdots, h_{n} \in H$ and $\epsilon>0$.

Then $\partial_{H} S$ exists.

Proof. Let $K$ be the class of all subsets $F$ of $S$ having properties 2.1 and 2.2. Let $K_{0}$ be a maximal decreasing chain in $K$, regarded as ordered by inclusion. This $K_{0}$ exists by Zorn's lemma. Let $F_{0}$ be the intersection of all members of $K_{0} . F_{0}$ is closed, and also satisfies 2.2 since for $h \neq 0$, the set $\{s ; h(s)=\max h\}$ (where $\max$ is always intended to refer to the maximum value of the function on $S$ ) intersects every $F$ in $K_{0}$ and hence intersects $F_{0}$. It remains to prove that $F_{0}$ satisfies 2.3 (with $F=F_{0}$ ). Suppose $B$ is closed in $S$ and does not contain $F_{0}$. Then some point $s_{0}$ of $F_{0}$ has a neighborhood $V$ defined as in 2.43 which does not meet $B$. Then $F_{0}-V$ being closed does not satisfy 2.2 . Hence $\max g$ is greater than $\sup g(s)$ for $s$ in $F_{0}-V$. By taking $h$ as a suitable power of $g$, we can obtain

$$
\max _{s \in F_{0}-V} h(s)<\frac{\epsilon \max h}{\max h_{1}+\cdots+\max h_{n}+1} .
$$

Let $s \in F_{0}$. If $s \in V$ then $h(s) h_{i}(s)<\epsilon \max h$. If $s \in F_{0}-V$ then again $h(s) h_{i}(s)<\epsilon \max h$, by 2.44 , and this is true for each $i$. Hence $\max$ $h h_{i}<\epsilon \max h$. Now suppose $h(t)=\max h$. Then $h(t) h_{i}(t)=h_{i}(t) \max h$ $<\epsilon \max h$ so that $t \in V$. Hence $h$ does not attain its $\max$ on $B$ at all. Thus 2.3 holds, and the theorem is proved.

2.5 Corollary. Let the hypothesis of 2.4 hold. Then $s_{0} \in \partial_{H} S$ if and only if for every neighborhood of $s_{0}$ there is an $h$ in $H$ which attains its max only in that neighborhood.

The "only if" was demonstrated above. The "if" part follows from 2.3.

3. Applications. Let $A$ be an algebra of continuous complex-valued functions vanishing at infinity on a locally compact space, with the 
property that for $s \neq t$ there is an $f$ in $A$ such that $f(s) \neq f(t)$. The theorem of Silov [III] says that then $\partial_{A} S$ exists. It may be of interest to prove that 2.4 really does generalize Silov's theorem. To this end, compactify $S$ with a point $\infty$ obtaining $S^{*}$. Let $B$ be the class of all $\lambda+f, f \in A$ and $\lambda$ complex. Let $H$ be the class of all $|g|, g \in B$. Then 2.43 holds with $S$ replaced by $S^{*}$, since $B$ has a unit [I, 5G] and 2.41, 2.43 are obvious. Let $F=\partial_{H} S^{*}-\{\infty\}$. First of all, every element of $A$ does attain its maximum modulus on $F$. Second, consider a closed subset $F_{1}$ of $S$ which does not include $F$. Then $F_{1} \cup\{\infty\}$ does not include $\partial_{H} S^{*}$, hence there is a $g$ in $B$ which does not attain its maximum modulus on the former set.

By scalar multiplication and suitable potentiation one can arrange that

$$
\begin{gathered}
\max _{s \in F_{1} \cup\{\infty\}}|g(s)|<1, \\
3<\max _{s \in S^{*}}|g(s)| .
\end{gathered}
$$

Let $g=\lambda+f, f \in A$. Then $|\lambda|<1$ because $f$ vanishes at $\infty$, whence $\max _{s \in F_{1}}|f(s)|<1+|\lambda|<2$, by 3.1. On the other hand, $|\lambda|<1$ together with 3.2 shows that $\max |f|>2$, whence $f$ does not attain its maximum modulus on $F_{1}$. Thus $F$ satisfies the definition of $\partial_{A} S$.

For later reference we sum this up briefly as follows.

3.3 The Šilov boundary for $A$ (with no unit) is obtained by deleting the point at infinity from the Silov boundary for $A$-with-unit-adjoined.

The fact that 2.4 does not require two algebraic operations makes possible another application. Let $L$ be a convex topological linear space, and let $S$ be a weakly compact subset of $L$. Let $\bar{L}$ be the class of linear continuous (possibly complex) functionals on $L$. Let $H$ be the class of functions $h=\left|e^{\lambda+f}\right|, f \in \bar{L}$, and $\lambda$ any complex constant. It is not hard to see that the sets of the form 2.43 with $\epsilon=1$ form a basis for the weak topology in $L$, and $2.41,2.42$ obviously hold. The set $\partial_{H} S$ is what has been called the $T$-frontier of $S$ by Milman [IV] in the case of Banach spaces. It can also be seen that it is the weak closure of the set of extreme points of the closed convex hull of $S$ (supposing that hull to be compact), by generalizing the argument presumably used by Milman.

4. Examples. Let $T$ be a compact Hausdorff space. Let $G$ be a group of homeomorphisms of $T$ that acts transitively. Let $A$ be a complete subalgebra, with unit, of $\subseteq(T, C)$ ( $C=$ complex numbers) with the obvious ring structure and norm, and satisfying:

$$
4.01 \text { if } t_{1} \neq t_{2} \text { then } f\left(t_{1}\right) \neq f\left(t_{2}\right) \text { for some } f \text { in } A \text {, }
$$


4.02 if $f \in A$ and $x \in G$ then $f x \in A$ where $(f x)(t)=f(x(t))$.

Now 4.01 shows that $T$ can be imbedded in the space $S$ of maximal ideals ${ }^{1}$ of $A$, by sending $t$ into $s$ if $f(s)=f(t)$ for all $f$ in $A$. We may therefore regard $A$ as a set of functions on $S$, and regard $T$ as a subset of $S$.

4.1 Theorem. Under the preceding assumptions, $T=\partial_{A} S$.

Proof. Every $f$ in $A$ attains its maximum modulus on $T$, whence $\partial_{A} S$ is contained in $T$. From 4.02 we see that $G$ induces automorphisms in $A$ which induce homeomorphisms of $S$ under which $\partial_{A} S$ is surely invariant. The induced homeomorphisms agree on $T$ with the way $G$ is supposed to act on $T$. Now $G$ is transitive on $T$, and $\partial_{A} S$ is a nonvoid invariant subset of $T$. Hence $\partial_{A} S=T$, as asserted.

Many specific examples of this sort of algebra can be obtained as follows.

Let $\Gamma$ be a discrete abelian group, and let $\pi$ be such a subset of $\Gamma$ that

$4.21 \alpha, \beta \in \pi$ implies $\alpha \beta \in \pi$ (multiplicative notation),

4.22 the neutral element 1 of $\Gamma$ belongs to $\pi$,

$4.23 \Gamma$ is the smallest subgroup containing $\pi$.

Now let $G=\{x, y, \cdots\}$ be the character group of $\Gamma$, and regard $\Gamma$ as functions on $G$. Let $A$ be closed subalgebra of $\mathbb{E}(G, C)$ generated by $\Gamma$ with the usual norm $\|f\|=\max |f(x)|, x \in G$. The group to act on $G$ shall be $G$ itself, acting as translations. Then $4.01,4.02$ are satisfied. Hence $G=\partial_{A} S$ where $S$ is the space of maximal ideals of $A$.

The special case in which $\Gamma$ is linearly ordered will be treated in detail in another paper.

The most important case of all this is when $\Gamma$ is the integers and $\pi$ is the set of non-negative integers. One arrives then at the classical theory of continuous functions on the unit disc which are regular in the interior. See Loomis [I, p. 81, Remark].

5. Measures on $\partial_{H} S$ representing points of $S$. We are about to generalize some of the following well-known facts.

Let $P(s, \theta)$ be Poisson's kernel, where $s$ is complex and $|s|<1$, and $\theta$ is the angle of the variable boundary point of the circle $|z|=1$. Then $m_{s}(d \theta)=(1 / 2 \pi) P(s, \theta) d \theta$ gives a measure on that circle such that if $f(z)$ is continuous for $|z| \leqq 1$ and regular for $|z|<1$ (or perhaps merely the real part of a regular function), then $f(s)=\int f\left(e^{i \theta}\right) m_{s}(d \theta)$, integration being the over the boundary.

We shall construct, for each $s \in S$ (notation of 2.4), a regular measure $m_{s}$ on, and supported by, $\partial_{H} S$, "representing" the point $s$. The

${ }^{1}$ Or rather, as we prefer to take it, the space of homomorphisms of $A$ onto $C$, and we shall write, somewhat ambiguously, $f(s)$ for the value of $s$ at $f$. 
"representation" is however not such that $h(s)=\int h(x) m_{s}(d x)$ (integration being over $\left.\partial_{H} S\right)$ for $h \in H$, but rather this: if $g$ and $g^{-1}$ both belong to $H$ then $\log g(s)=\int \log g(x) m_{s}(d x)$. In the special case of the regular functions, i.e., $H$ the class of all $|f|$ where $f$ is continuous on the disc and regular inside, the familiar result is easily recovered, as follows. Form $g=\left|e^{f}\right|$; then $g$ and $g^{-1}$ both belong to $H$ so that by the result on $\log g$, we have $R f(s)=\int R f(x) m_{s}(d x)$, from which the desired result can be concluded.

The hypothesis for this section will be now stated once for all.

$5.01 S$ is a topological space and $H$ is a class of real-valued nonnegative continuous functions on $S$ such that

5.02 If $h, j \in H$ then $h j \in H$.

5.03 Every element of $H$ attains its maximum on some fixed compact Hausdorff subset $B$ of $S$.

5.04 There is an element $k$ in $H$ which is a positive constant less than 1 , on $B$.

It is clear that if $2.41-2.43$ hold, then $B$ exists. However, we have no use for 2.43 in this section.

We begin by introducing a linear space of continuous real functions on $S$.

Let $E$ be the class of all real linear combinations of functions $\log g$, where $g$ and $g^{-1}$ both belong to $H$.

5.1 Lemma. Let $h \in H, u \in E$, and suppose $\mu$ is a non-negative real number. Suppose $\mu \log h \leqq u$ on $B$. Then $\mu \log h \leqq u$ on $S$.

Proof. Let $v=-u$; then we have $\mu \log h+v \leqq 0$ on $B$. Let us assume that there is an $s$ in $S$ such that $\mu \log h(s)+v(s)>\epsilon>0$. (The ensuing contradiction will prove the theorem.) Select a positive integer $p$ exceeding $-\epsilon^{-1} \log k$. Suppose $v=\sum \lambda_{i} \log g_{i}$ where the $g_{i}$ have inverses in $H$. Then

$$
\mu \log h+\sum \lambda_{i} \log g_{i}+\frac{1}{p} \log k<0
$$

on $B$,

and

$$
\mu \log h(s)+\sum \lambda_{i} \log g_{i}(s)>\epsilon .
$$

Now find rational approximations $m / n, m_{i} / n$ for $\mu, \lambda_{i}$ respectively (with common positive denominator $n$ ) such that if the Greek letters in 5.12 and 5.13 are replaced by the approximations, then the inequalities still hold. It follows that

$$
h^{p m} k^{n}\left(g_{1}^{m_{1} g_{2} m_{2}} \cdots\right)^{p}<1
$$


whence the same is true on all of $S$ and so

$$
\frac{m}{n} \log h(s)+\sum \frac{m_{i}}{n} \log g_{i}(s)+\frac{1}{p} \log k<0 .
$$

Considering the rational approximation of 5.13 , we see that $-p^{-1} \log k>\epsilon$ which contradicts the choice of $p$.

5.2. Theorem. Let 5.01-5.04 hold. Let $s$ in $S$ be given. Then there is a regular Baire measure $m_{8}$ defined on $B$ such that

$$
\log g(s)=\int_{B} \log g(x) m_{s}(d x)
$$

whenever both $g$ and $g^{-1}$ belong to $H$.

Proof. For $u$ in $E$ let $u^{\prime}$ be the function $u$ with domain restricted to $B$. This gives a linear mapping of $E$ onto a subspace $E^{\prime}$ of $\mathbb{(}(B, R)$. For $s$ in $S$ define $J\left(u^{\prime}\right)=u(s)$. Taking $h=k$ and $\mu=0$ shows that $J$ is non-negative, and it is clearly linear. Suppose now that $u \leqq 1$ on $B$. Let $\mu=-(\log k)^{-1}$, which is positive, and we have $\mu \log k \leqq-u$. Applying 5.1, we obtain $u(s) \leqq 1$. This shows that $J$ has a bound not greater than 1. A well-known argument now gives an integral representation for $J(s)$ in terms of a measure $m_{s}$ on $B$ and this is the measure $m_{s}$ on $B$ such that $u(s)=\int_{B} u(x) m_{s}(d x)$. This is evidently the measure we promised to construct. It is not necessarily unique, as the following example, involving merely regular functions of 2 complex variables, will show.

Let $\Gamma$ be $J^{2}$, where $J$ is the group of integers, and let $\Pi$ be the class of pairs of non-negative integers. This sets in motion the train of ideas presented in $\S 4$. The space $S$ of maximal ideals of $A$ is (homeomorphic to) the class of pairs $(\lambda, \mu)$ where $\lambda, \mu$ are complex and $|\lambda|$, $|\mu| \leqq 1$. By the maximum modulus principle, $\partial_{A} S$ is the class of pairs $(\lambda, \mu)$ where $|\lambda|=|\mu|=1$ which is the character group of $J^{2}$. Reference to 4.1 produces the same result. For $f \in A, f(\lambda, \mu)=a_{0}+a_{1} \lambda$ $+b_{1} \mu+\cdots$, , we surely have $f(0,0)=\int_{G} f(\lambda, \mu)|d \lambda||d \mu|$ where $\mid$ refers to the usual (Haar measure) of the circle group. Thus the Haar measure of the torus $G$ can be used to represent the point $(0,0)$ of $S$. But the 1-dimensional Haar measure of certain 1-dimensional closed subgroups will produce the same representation. In fact, let $\lambda=\nu^{m}, \mu=\nu^{n}$ (where $|\nu|=1$ and $m, n$ are positive integers) parametrize a subgroup $G_{1}$ of $G$. Then $\int f\left(\nu^{m}, \nu^{n}\right)|d \nu|=f(0,0)$ (integration being over the circle group).

6. Further properties of the measures. From a theorem of Szegö 
[II] one knows that for $f$ regular in the disc and continuous on the boundary, the function $\log |f|$ is integrable (unless $f=0$ ) over the boundary of the disc, and in any case,

$$
\log |f(0)| \leqq \int \log |f|
$$

integration being over the boundary, with the usual measure divided by $2 \pi$. For each interior point of the disc there is an analogous inequality, involving the measure representing the point in question. We shall generalize this proposition. It will actually be a consequence of findings on the totality of values of $I_{s}(\log h)$, where $I_{s}$ runs through all possible extensions of $J_{s}$. In the classical case there is only one extension of $J_{s}$. If the reader were to deflate the following considerations to this special case, he would obtain a proof of 6.01 rather different from Szegö's, since it makes no use of the group structure of the boundary of the disc.

The hypothesis 5.01-5.04 will be assumed for this section just as for the last. The set $E$ is that defined in the last section.

Now let $l$ be any extended-real-valued (meaning that $\pm \infty$ are permitted as values) function defined on $B$. Then another extendedreal-valued function, defined on all of $S$, can be constructed thus:

$$
l^{-}(s)=\inf _{u \in E, u \geqq \operatorname{lon} B} u(s)
$$

where we let the inf be $+\infty$ if there are no such $u$. A dual function $l_{-}$can be defined, using a sup, or we may simply define $l_{-}=-(-l)^{-}$.

Properties of this sort of prolonged upper envelope are listed in the following.

6.2 TheOREM. The function $l^{-}$is upper semicontinuous, and

$$
l_{-} \leqq l \leqq l^{-} \text {on } B,
$$

$$
l_{1} \leqq l_{2} \text { on } B \text { implies } l_{\overline{1}} \leqq l_{2}^{-} \text {on } S \text {, }
$$

$$
\left(l_{1}+l_{2}\right)^{-} \leqq l_{1}^{-}+l_{2}^{-} ;(c l)^{-}=c l^{-} \text {for } c \geqq 0 ;
$$

6.24 for $l=\log h, h \in H$, the inequality $\log h \leqq(\log h)$ - holds on all of $S$.

We shall consider only the proof of 6.24 . Suppose $u \geqq \log h$ on $B$. Then $u \geqq \log h$ on $S$ by 5.1. Taking the inf of these $u$, we get 6.24 .

In the definition (6.1) of $l^{-}(s)$ we consider the class of all $\sum \lambda_{i} \log g_{i}$ which dominate $l$ on $B$. We note next that actually only sums of one term need to be considered.

6.25 Theorem. Suppose that $k^{-1} \in H$, so that $\log k \in E$. Then 


$$
l^{-}(s)=\inf \left[\frac{1}{n} \log g(s)\right]<\infty
$$

where $(g, n)$ runs through all $g \in H$ such that $g^{-1} \in H$ and $g \geqq e^{n l}, n$ being a positive integer.

Proof. Let the right-hand side of 6.25 be called $a$. Then surely $a \geqq l^{-}(s)$. Now let $l^{-}(s)<b$. Then there is a $u \in E$ such that $u \geqq l$ on $B$ and $u(s)<b$. Since $\log k$ may be assumed present in $u$, we can alter its coefficient a bit and get $u>l$ on $B$. With this elbow room we can replace all coefficients by rational numbers, and obtain a relation like $\sum n_{i} \log g_{i}>n l, \sum n_{i} \log g_{i}(s)<n b$ where the $n_{i}$ and $n$ are positive integers. Let $g=g_{1}^{n_{1}} g_{2}^{n_{2}} \ldots$. Then $(1 / n) \log g(s)<b$, so that $a<b$. Thus $a \leqq l^{-}(s)$.

We want now to extend $J_{s}$ to all of $\mathfrak{C}(B, R)$ and we want to see what are the possible values of the extension $I_{s}$ for a fixed $l \in \mathbb{E}(B, R)$.

6.3 Theorem. Let $l \in \mathfrak{S}(B, R)$; let $s \in S$; and suppose $l_{-}(s) \leqq j \leqq l^{-}(s)$. Then

$6.31 u_{1}+\lambda l \leqq u_{2}+\mu l$ implies $u_{1}(s)+\lambda_{j} \leqq u_{2}(s)+\mu j$ for every pair of elements $u_{1}, u_{2}$ of $E$;

6.32 by setting $J^{\prime}(u+\lambda l)=u(s)+\lambda j$ one obtains a linear, non-negative extension to the linear space of the $u+\lambda l$ of the functional $J_{s}$, and its bound is still 1;

6.33 all linear non-negative extensions $I_{s}$ of $J_{s}$ from $E$ to $\mathbb{S}(B, R)$ can be obtained in this way.

Proof. We consider the case $\lambda<\mu$ in 6.31, leaving the others to the reader. Then $u=(\mu-\lambda)^{-1}\left(u_{1}-u_{2}\right) \leqq l$ on $B$, so that $(\mu-\lambda)^{-1}\left(u_{1}(s)\right.$ $\left.-u_{2}(s)\right) \leqq l_{-}(s) \leqq j$, from which the desired inequality follows.

Part 6.32 follows easily from 6.31. Part 6.33 follows from the fact that $l_{-}(s) \leqq I_{s}(l) \leqq l-(s)$ for all such extensions of $J_{s}$.

6.34. CoRollary. If every $J_{s}$ has only one non-negative linear extension to $C(B, R)$ or if (which is assuming at least as much) $E$ is dense in $C(B, R)$, then there is a linear, order-preserving mapping $l \rightarrow l^{*}$ of $C(B, R)$ to $C(S, R)$ such that $l^{*}$ is an extension of $l$, and such that if $l$ is the restriction of $u \in E$ to $B$, then $l^{*}=u$.

For the proof, let $l^{*}=l_{-}$. By $6.3, l^{*}=l^{-}$. Finally, 6.2 insures that $l^{*}$ has all the properties stated.

This theorem (6.34) constitutes a kind of general solution to Dirichlet's problem. We shall not pursue this topic, and return to the question of what values $I_{s}(\log h)$ can have, for $h$ in $H$ but having possible zeros on $B$ so that 6.3 does not apply. 
6.4. Theorem. Let $h \in H$, and $s \in S$, and suppose $k^{-1} \in H$ (see 5.04). Then the totality of values of $I_{s}(\log h)$, where $I_{s}$ ranges over all extensions to $C(B, R)$ of $J_{s}$, is an interval possessing at least its upper end point, which is $(\log h)-(s)$, plus possibly the point $-\infty$. In the latter event, the point $-\infty$ may or may not be the lower end point.

Proof. The set of measures $\left\{m_{s}\right\}$ representing the various $I_{s}$ is clearly convex, and so therefore is the set of values $I_{s}(\log h)$ insofar as they are finite. Therefore, except for the detail about $-\infty$ which we shall settle by an example, the main thing to prove is the identity of the upper end point. It is of course clear as in 6.2 that $I_{s}(\log h)$ $\leqq(\log h)-(s)$. We shall now construct an $I_{s}$ such that $I_{s}(\log h)$ $\geqq(\log h)^{-}(s)$.

Let $a$ be a real positive number. Let $j_{a}=(\log (h+a))-(s)<\infty$ by 6.25. By 6.3 there exists an extension $I_{a}$ of $J_{s}$ such that $I_{a}(\log (h+a))$ $=j_{a}$. These $I_{a}$ all belong to the unit ball of the conjugate space, $\mathfrak{C}(B, R)^{-}$, and hence cluster weakly at some $I$ of the unit ball, as $a \rightarrow 0$.

Let $b \geqq a$. Then, since $\log h \leqq \log (h+a) \leqq \log (h+b)$, one has $(\log h)^{-}(s) \leqq j_{a} \leqq I_{a}(\log (h+b))$. For fixed $b$, the last term clusters at $I(\log (h+b))$ as $a \rightarrow 0$. Hence $(\log h)-(s) \leqq I(\log (h+b))$. As $b \rightarrow 0$, $\log (h+b)$ tends monotonely to $\log h$ on $B$ (in fact, on $S$ ), on which $I$ is represented as an integral, so $(\log h)^{-}(s) \leqq I(\log h)$. This is the desired inequality. What we have proved means that $\log h$ is summable- $I$ if and only if $(\log h)-(s) \neq-\infty$.

From 6.24 and 6.4 we have the following:

6.41 CoROLlaRy. If $J_{s}$ has only one extension $I_{s}$, and $m_{s}$ is the representing measure, then

$$
\log h(s) \leqq \int_{B} \log h(x) m_{s}(d x) .
$$

The following rather trivial example shows that our assumptions permit us to say nothing more about the values of $I_{s}(\log h)$ in general. Let $S=s_{1}, s_{2}, s_{3}$ be a set of three points, and let $H$ be the class of all non-negative constant functions on $S$ (to satisfy 5.04 ) plus the set of all non-negative functions which vanish for at least one point (to satisfy 2.43). Actually, all axioms made above are satisfied. Clearly $\partial_{H} S$ is $S$ itself, and $E$ is one-dimensional. Hence any measure on $S$ with $m(S)=1$ represents each of the three points. Let $h\left(s_{1}\right)=0$, $h\left(s_{2}\right)=\lambda, h\left(s_{3}\right)=\mu$ with $0 \leqq \lambda \leqq \mu$. Then the set of values for $I(\log h)$ is the closed interval $[\log \lambda, \log \mu]$ plus $($ if $\lambda \neq 0$ ) the point $-\infty$. Notice that one may have $-\infty \neq I(\log h)<\log h(s)$. 
The final remarks of this section are devoted to showing that the theorems proved above constitute a generalization of Szegö's inequality 6.01 .

We return to the classical example already referred to at the end of $\$ 4$, which is the Banach algebra $A$ of functions continuous on the $\operatorname{disc} S=\{|\lambda| \leqq 1\}$ and regular for $|\lambda|<1$. We let $H$ be the class of $|f|$, with $f \in A$. Obviously $\partial_{H} S$ is the ordinary boundary $B=\{|\lambda|=1\}$. The set $E$ (regarded as continuous functions on $B$ ) surely contains all real parts $R f$ for $f \in A$ since $\log \left|e^{f}\right|=R f$ and $\left|e^{f}\right|$ has its inverse $\left|e^{-f}\right|$ in $H$. Since every trigonometric polynomial is the boundary value of some polynomial $f(z)$, the $\{R f\}$ and thus $E$ is dense in $C(B, R)$ so that each $s \in S$ has precisely one representing measure on $S$, which is now to be identified as that given by Poisson's integral. Let us consider merely $s=0$. By Cauchy's formula, if $f \in A$ then $f(0)=(2 \pi)^{-1} \int_{0}^{2 \pi} f\left(e^{i \theta}\right) d \theta=\int_{B} f$ with a suitable normalization of the measure. Now let $l=\log \left|e^{f}\right|$. Then $l \in E$, so that Cauchy's formula gives $l(0)=\int_{B} l$. Thus the Haar measure on $B$ does give the unique representing measure, since the class $\{R f\}$ is large enough to define it. Now let $l=\log |f|, f \in A$. Then $\log |f(0)| \leqq(\log |f|)^{-(0)}$ by 6.24, and $(\log |f|)^{-}(0) \leqq \int_{B} \log |f|$, by 6.4 . This gives 6.01 . The example $f(\lambda)=\lambda$ exhibits a phenomenon that should not be overlooked (cf. Jensen's formula).

7. Noncompact spaces of maximal ideals. Suppose we have a commutative Banach algebra $A$ and $S$ is its space of maximal ideals, and $H$ is the class of functions $|f|, f$ in $A$, regarded as defined on $S$. Then $5.03,5.04$ will not be satisfied unless $A$ has a unit. Hence we adjoin a unit, which adds a point at infinity to $S$, while 3.3 says that precisely the same thing happens to the Silov boundary $B$. Now the representing measures will a priori require for their support the set $B^{*}=B \cup\{\infty\}$ in $S^{*}=S \cup\{\infty\}$. However, the point $\infty$ may be regarded as of measure 0 in every case, since all the $f$ from $A$ vanish there.

We shall formulate the result of applying these considerations to 5.2 and 6.4 .

7.1 Theorem. Let $A$ be a commutative Banach algebra. Let $S$ be the space of maximal ideals. Let $B$ be the Šilov boundary. We regard the elements of $A$ as functions on $S$. Let $s$ be any point of $S$. Then there is a regular Baire measure $m_{s}$ defined on $B$ such that

7.11

$$
\begin{aligned}
m_{s}(B) & \leqq 1, \quad \text { for every } f \text { in } A ; \\
\int_{B} f(x) m_{s}(d x) & =f(s) \quad
\end{aligned}
$$


and for each particular $f$ the measure may be so chosen that (moreover)

$$
\int_{B} \log |f(x)| m_{8}(d x) \geqq \log |f(s)| .
$$

Naturally, if for some reason there is for some $s$ only one measure satisfying 7.12 , then 7.13 holds for that measure.

\section{BIBLIOGRAPHY}

I. L. H. Loomis, An introduction to abstract harmonic analysis, New York, 1953.

II. G. Szegö, Über die Randwerte einer analytischen Funktion, Math. Ann. vol. 84 (1921) pp. 232-244.

III. I. Gelfand, D. Raikov, and G. Šilov, Commutative normed rings, Uspehi Matematiceskih Nauk N.S. vol. 2 (1946) pp. 48-146. (In Russian).

IV. D. Milman, Characteristics of extremal points of regularly compact sets, Doklady Akademia Nauk SSSR N.S. vol. 57 (1947) pp. 119-122 (In Russian); Mathematical Reviews vol. 9 (1948) p. 192.

University of California, Los Angeles

\section{FAMILIES OF CURVES}

\section{S. STEIN}

Amasa Forrester in [1] proved the following theorem of a mixed Euclidean and topological character. If $\phi$ is a continuous map without fixed points on the Euclidean $n$-sphere such that $\phi^{2}$ is the identity, then the chords $P \phi(P)$ for all points $P$ of the sphere completely fill the interior of this sphere.

The object of this note is to generalize this theorem to a purely topological statement.

First we recall the definition of retract. If $B C A$ are two spaces, then $B$ is a retract of $A$ if there is $r: A \rightarrow B$ which leaves fixed all points of $B$. (If $X$ and $Y$ are spaces the symbol $f: X \rightarrow Y$ shall denote a continuous map from $X$ to $Y$.)

Let $I$ denote the unit interval. If $F: B \times I \rightarrow A$ and $t \in I$, define $F_{t}: B \rightarrow A$ by $F_{t}(b)=F(B, t)$ for all $b \in B$.

ObServation. If $F: B \times I \rightarrow A$ and if $B$ is a retract of $A$ by the map $r$ and if $p, q \in I$, then $r F_{p}$ is homotopic to $r F_{q}$.

In fact such a homotopy is provided by $G: B \times I \rightarrow B$ defined by

Presented to the Society, November 28, 1953; received by the editors October 26, 1953 and, in revised form, February 28, 1954. 